\title{
Das Institut für Plasma- und Lasertechnik an der TFH Wildau - Entwicklung und Kooperation mit anderen Einrichtungen
}

\author{
Harald Beyer, Andreas Harendt, Karin Huget, Tobias Koschlig, \\ Siegfried Rolle, Werner Ross, Hans Volkmann
}

Die Plasmatechnik gehört mittlerweile zu den Schlüsseltechnologien des nachhaltigen Wachstums und der Bewältigung des Strukturwandels der deutschen Wirtschaft. Plasmaverfahren sind zur Voraussetzung für die Herstellung von Chips für Hochleistungscomputer geworden. Mit Plasmatechnologien werden Werkstoffoberflächen veredelt und Materialien mit neuen Eigenschaften erzeugt. Es gibt Plasmalampen, die ohne Verwendung von Quecksilber hohe Lichtausbeuten und gute Farbwiedergabe erreichen. Auch bei der Entsorgung toxischer Stoffe und von Abfällen kommen Plasmatechnologien zum Einsatz. Das Bundesministerium fuir Bildung und Forschung (BMBF) hat in einer Broschiire die Anwendung und Forschung auf dem Gebiet der Plasmatechnik in Deutschland zusammengestellt [1]. In Deutschland wird jährlich eine Industrieproduktion von 45 Mio. $€$ durch die Plasmatechnologie erst ermöglicht. Vor diesem Hintergrund soll die Entwicklung der Forschung und Ausbildung auf dem Gebiet der Plasmatechnik an der TFH Wildau beschrieben werden.

Die Wurzeln des Institutes reichen weit in die Geschichte der deutschen Plasma- und Lasertechnik zuruick. Die Entwicklung der Plasmaphysik wurde vor dem zweiten Weltkrieg hauptsächlich durch Grundlagenuntersuchungen zu Fragen der Leitung des elektrischen Stromes in Gasen, aber auch durch Fragestellungen aus der Lichtquellenindustrie angetrieben. Zentren waren Eindhooven mit der Phillips Forschung und Berlin mit der Osramstudiengesellschaft. Hier war auch Robert Rompe tätig, der in dieser Zeit bedeutende Beträge zur Plasmaphysik geliefert hat. In dieser Zeit wurden die Ellenbaas-HellerGleichung aufgestellt, die Saha-Gleichung und die Funken-Gesetze (Rompe, Weizel) formuliert [2]. Mit der Teilung Deutschlands nach Ende des Krieges wurde aus dem Osramwerk an der Oberbaumbrïicke das Berliner Glïhlampenwerk (BGW). Später erfolgte die Eingliederung des BGW in das DDR-Kombinat NARVA. Aus der Osramstudiengesellschaft wurde das Institut für Strahlungsquellen in Ostberlin.

Als Rompe begann in Berlin in einem Großinstitut Festkörper- und Plasmaphysik zu vereinigen, wurde auch das Institut für Strahlungsquellen dem Zentralinstitut für Elektronenphysik der Akademie der Wissenschaften der DDR (ZIE) angeschlossen. Ein weiteres Zentrum der Plasmaphysik war Jena. Dort entstand unter Leitung von Max Steenbeck ein Forschungsinstitut mit plasmaphysikalischer Ausrichtung. Die Plasmaphysik beschäftigte sich weiterhin mit Lichtquellen und der Wechselwirkung von elektromagnetischen Wellen mit Plasmen. Zur da- maligen Zeit schien die gesteuerte Kernfusion ähnlich leicht realisierbar wie die friedliche Nutzung der Kernspaltung. In Jena wurde ein Plasmafokusexperiment aufgebaut und auch in Spreeau bei Berlin wurde fuir die Kernfusion geforscht. Doch bald musste man erkennen, dass die Kernfusion nur mit erheblichem Aufwand bis zur technischen Nutzung gebracht werden würde. Die Kernfusion wurde daher nur noch in Kooperation mit internationalen Einrichtungen, maßgeblich dem Kurtschatow-Institut in Moskau, betrieben. Hauptsächlich wurden Experimente zur Untersuchung der wandnahen Plasmen und der Plasma-Wandwechselwirkung in Berlin konzipiert und in Tokamak-Experimenten wie T10 und T15 in Moskau durchgefuihrt.

Das Zentralinstitut fuir Elektronenphysik, dem nach Steenbecks Tod das Jenaer Institut angegliedert wurde und zu dem auch die unter Rudolf Seeliger beruihmt gewordene Schule in Greifswald gehörte, bearbeitete die Plasmaphysik in ihrer ganzen Breite. Rudolf Seeliger wurde durch Johannes Stark nach Greifswald berufen. Seeliger hatte mit einer preisgekrönten Dissertation bei Arnold Sommerfeld in München auf sich aufmerksam gemacht und dann uiber Jahrzehnte das wissenschaftliche Profil in Greifswald geprägt [3, 4].

Werner Neumann verfasste sein von Rompe und Steenbeck herausgegebenes Buch über die Plasmaspektroskopie [5], das noch heute, obwohl sich die experimentelle Technik rasant weiter entwickelt hat, ein viel genutztes Standardwerk der Plasmaphysik geblieben ist. Harald Güindel begann als Schüler von Neumann mit Untersuchungen zur Nutzung von spektralen Strahlungsverteilungen an einer Vielzahl von unterschiedlichen Metallen. Hintergrund der Arbeit war die Suche nach Zusätzen, um Farbwiedergabe und Lichtausbeute der Quecksilberhochdrucklampen zu verbessern. Wenig später publizierte er einen Artikel zur Erniedrigung der lonisierungsenergie [6]. In der amerikanischen Fachliteratur wurde der Effekt mit seinem Namen verbunden. Zusammen mit Werner Ross und Klaus Seeliger begann er mit der Entwicklung von Gaslasern. Die Anregung der Laser erfolgte mit einem nichtthermischen Impulsplasma. Besonders erfolgreich war die Entwicklung einer ganzen Familie von Stickstofflasern. Die Laser wurden als Pumpquelle für Farbstofflaser und für Bearbeitungsaufgaben im Mikrometerbereich genutzt und in den Akademiewerkstätten in gröBerer Stückzahl produziert.

In Jena beschäftigte man sich unter der Leitung von Willi Ebert mit Hochstrombogenentladungen in Edelgasen 


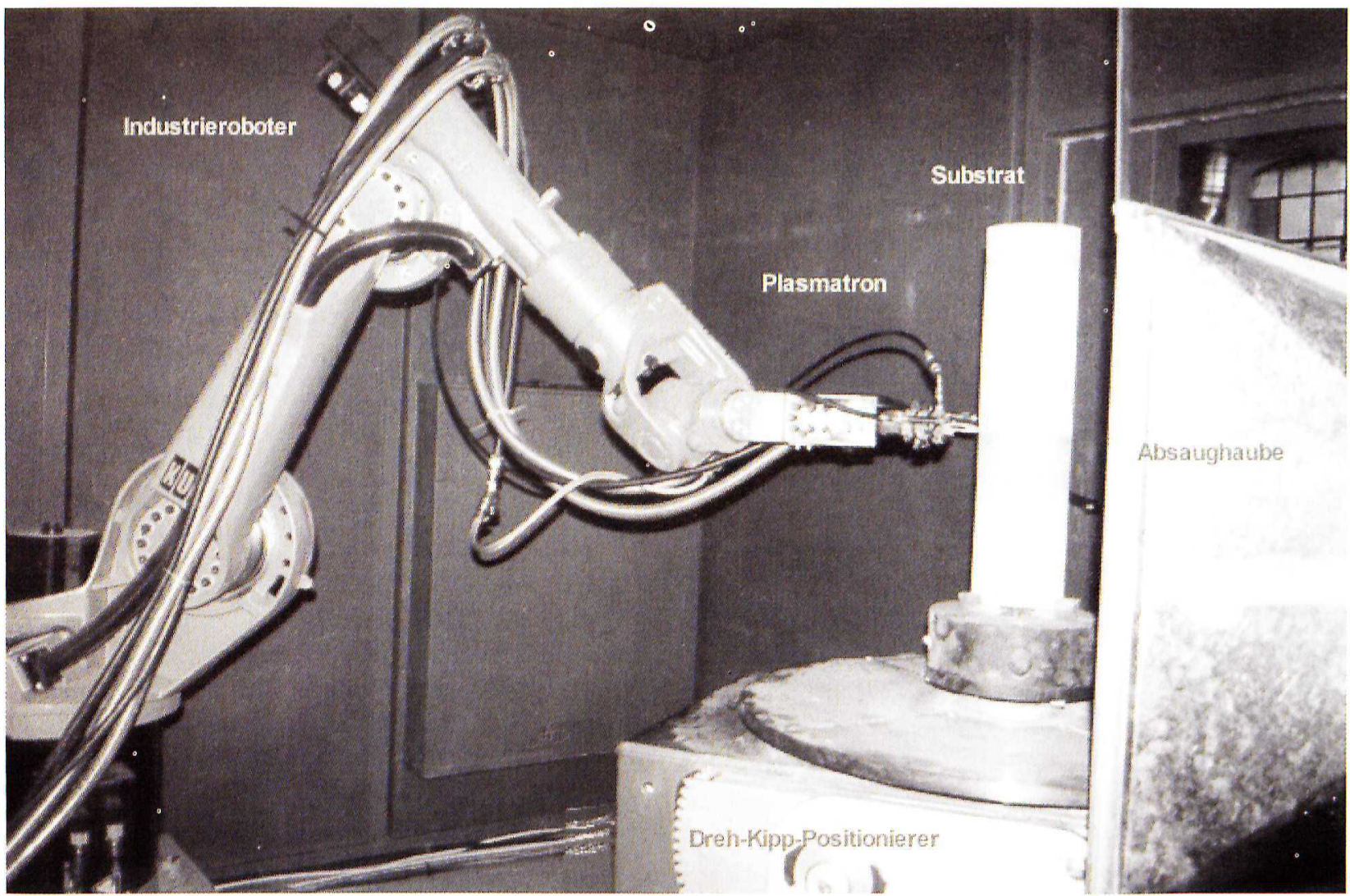

Plasmaspritzen mit Industrieroboter

und Hochfrequenzentladungen in Gold- und Kupferdämpfen-Forschungen, die sich in der Produktion der Edelgasionenlaser in den Carl Zeiss-Werken und bei den Metalldampflasern wiederfinden.

Große Beachtung fanden auch die Arbeiten von Klaus Günther und Rainer Radtke zu Natriumhochdruckentladungen fuir den Betrieb im Kilohertzbereich. Hier wurden Grundlagen für die Ablösung von Quecksilber in Entladungslampen fuir die Innenraumbeleuchtung gelegt. Bemerkenswert war auch der Aufbau des Plasmatechnikums durch Harry Erbs, der bei NARVA die Natriumhochdrucklampe zur Produktionsreife gefuihrt hatte. Das Technikum lieferte mit modernsten Technologien die Basis für experimentelle Arbeit des Instituts und war daruiber hinaus in der Lage, Vorserien und Kleinserien zu produzieren. Ein Beispiel ist eine Strahlungsquelle, die auf einem optisch dichten Impulsplasma beruht und mit einer Temperatur von $15000 \mathrm{~K}$ als Normalstrahler für die Spektroskopie im ultravioletten Spektralbereich Verwendung fand.

Die deutsche Wiedervereinigung brachte zwar das Ende der Akademie der Wissenschaften der DDR, aber nach der Evaluierung des ZIE durch eine Kommission des Wissenschaftsrates ergab sich, dass die große Mehrzahl der Forschungsprojekte in der gesamtdeutschen Forschungslandschaft eine Fortsetzung fand. Die fusionsorientierte Plasmaphysik des ZIE wurde an das Institut für Plasmaphysik der Max-Planck-Gesellschaft übernommen. Ein Teil der Mitarbeiter arbeitet in Greifswald am Aufbau des neuen Stellerators „Wendelstein 7X“ mit. Der andere Teil ist im Bereich Plasmadiagnostik in Berlin unter der Leitung von Gerd Fußmann tätig. In Berlin werden zwei große Experimente betrieben - eine Elektronenstrahlionenfalle zur Untersuchung hochangeregter Zustände von Ionen und ein Plasmagenerator, in dem mit einer Hohlkathodenbogenentladung Plasmabedingungen erzeugt werden können, wie sie in den Randschichten von Fusionsmaschinen auftreten.

In Greifswald wurde das Institut für Niedertemperaturplasmaphysik (INP) gegruindet. Es entstand aus dem Greifswalder Institutsteil des ZIE und Teilen des Bereiches Niedertemperatur Plasmaphysik aus Berlin. Die Aufbauphase des Institutes wurde von Johannes Conrads geleitet. Das Themenspektrum reicht von der Modellierung anwendungsnaher Plasmen über die Untersuchung reaktiver Molekuilplasmen, speziell in ihrer Wechselwirkung mit Festkörperoberflächen, bis hin zu Forschungen an neuartigen Plasmalichtquellen, die in Verbindung mit der Osramforschung durchgeführt werden, und Fragen der Plasmachemie.

In dem Zusammenhang ist interessant, dass eine Reihe von Mitarbeitern der NARVA-Forschung und von Mitarbeitern des ZIE, die an NARVA-Forschungsaufträgen mitgearbeitet haben, jetzt in der Osramforschung tätig sind. Dazu gehören u. a. Klaus Günther, der bis 1990 Forschungsdirektor bei NARVA war und Hans-Georg Kloss, der uiber viele Jahre auf den Gebiet der Hochdruckplasmen, wie sie in Gasentladungslampen zum Einsatz kommen, und der Plasmaspektroskopie gearbeitet hat.

Harald Gündel, der bis 1988 die NARVA-Forschung geleitet hat und danach Direktor des ZIE war, suchte den Kontakt zur 1991 neu gegründeten Technischen Fach- 
hochschule Wildau. Mit der Unterstuitzung des Gründungsrektors Wilfried Arlt und des Fachbereiches Physikalische Technik wurde 1993 das Institut für Plasmaund Lasertechnik (IPLT) an der TFH Wildau gegrüindet. Ziel war es, eine forschungs- und praxisnahe Studentenausbildung aufzubauen. Die Mitarbeiter des Institutes forschten im Rahmen von Projekten des Bundesministeriums für Forschung und Technologie (BMFT) an effizienten Anregungssystemen von Lasern durch Hochfrequenz- und Mikrowellenplasmen [7] und zu Grundlagen der nichtthermischen Plasmen bei Atmosphärendruck. Gündel begann mit der Ausarbeitung von Vorlesungen und Praktika zur Plasma- und Lasertechnik.

Nach seinem frühen Tod 1996 gelang es der Fachhochschule, das Institut weiterzufuihren. Die Leitung wurde von Siegfried Rolle übernommen. Den Mitarbeitern des Institutes gelang es, die von Gündel begonnenen Aktivitäten weiterzufuihren und auszubauen. So wird neben der Vorlesung zur Laserphysik auch eine Vorlesung zur Lasermesstechnik und zur Laseroberflächentechnik angeboten. Auch bei der Plasmatechnik sind Vorlesung und Praktikum mittlerweile fester Bestandteil der Ausbildung. Die Laborausruistung reicht von Lasersystemen uiber Diagnostik bis zu einer kommerziellen Plasmaspritzanlage.

Das Institut für Plasma- und Lasertechnik hat während seines Bestehens zu einer Reihe von Institutionen und Firmen, die auf dem Gebiet der Plasma- und Lasertechnik tätig sind, Kooperationsbeziehungen aufgebaut. Diese Kooperationsbeziehungen ermöglichen es der TFH Wildau, unmittelbar an modernen Forschungsvorhaben teilzuhaben und damit auch eine Studentenausbildung, die sich an Anforderungen von Forschung und Industrie orientiert, zu ermöglichen. An einigen ausge- wählten Beispielen wollen wir die Kooperationsbeziehungen näher darstellen.

Im Jahresbericht des Max-Planck-Institutes für Plasmaphysik für das Jahr 2000 wird über die Zusammenarbeit mit der TFH Wildau berichtet. Anlass war der Abschluss einer Forschungsarbeit für Joint Europeen Torus Undertaking (JET), einem größen Fusionforschungsexperiment in Großbritannien. Es ging dabei um Untersuchungen zur Heliumstrahldiagnostik der Plasmarandschicht $[8,9]$. Die Kooperation mit dem IPP begann bereits 1997 mit der gemeinsamen Entwicklung von laserbearbeiteten Teilen für Plasmasonden für das Experiment „Asdex“ und plasmagespritzten Bauteilen füir den Plasmagenerator „PSI 1“. Später wurden gemeinsame Untersuchungen zur Zweistrahllaserspeckleinterferometrie und zur laserinduzierten Fluoreszenz am „PSI 2"durchgeführt. Im Rahmen eines Praktikums am IPP nahm eine Studentin aus Wildau ein Röntgenspektrometer erfolgreich in Betrieb. Während der Laserausbildung erhalten die Studenten der TFH die Möglichkeit, die derzeit modernsten Lasersysteme zur Plasmadiagnostik kennen zu lernen.

Auch mit dem Institut für Niedertemperaturplasmaphysik (INP) werden enge Beziehungen gepflegt. Gemeinsam wurden laserbearbeitete Titanringe entwickelt, die zum Aufbau von Entladungsgefäßen benötigt werden. Im Bereichskolloquium des INP wurde über die Untersuchungen an der TFH Wildau zum Einsatz einer Koronaentladung fuir die Beseitigung von Schadgasen berichtet. Auf dem Gebiet der Molekülspektroskopie gab es einen interessanten Erfahrungsaustausch. Im INP wurde ein Programmpaket zur Bestimmung von Gastemperaturen aus Stickstoffmolekuilspektren, das von Mitarbeitern des IPLT mitentwickelt wurde, genutzt.

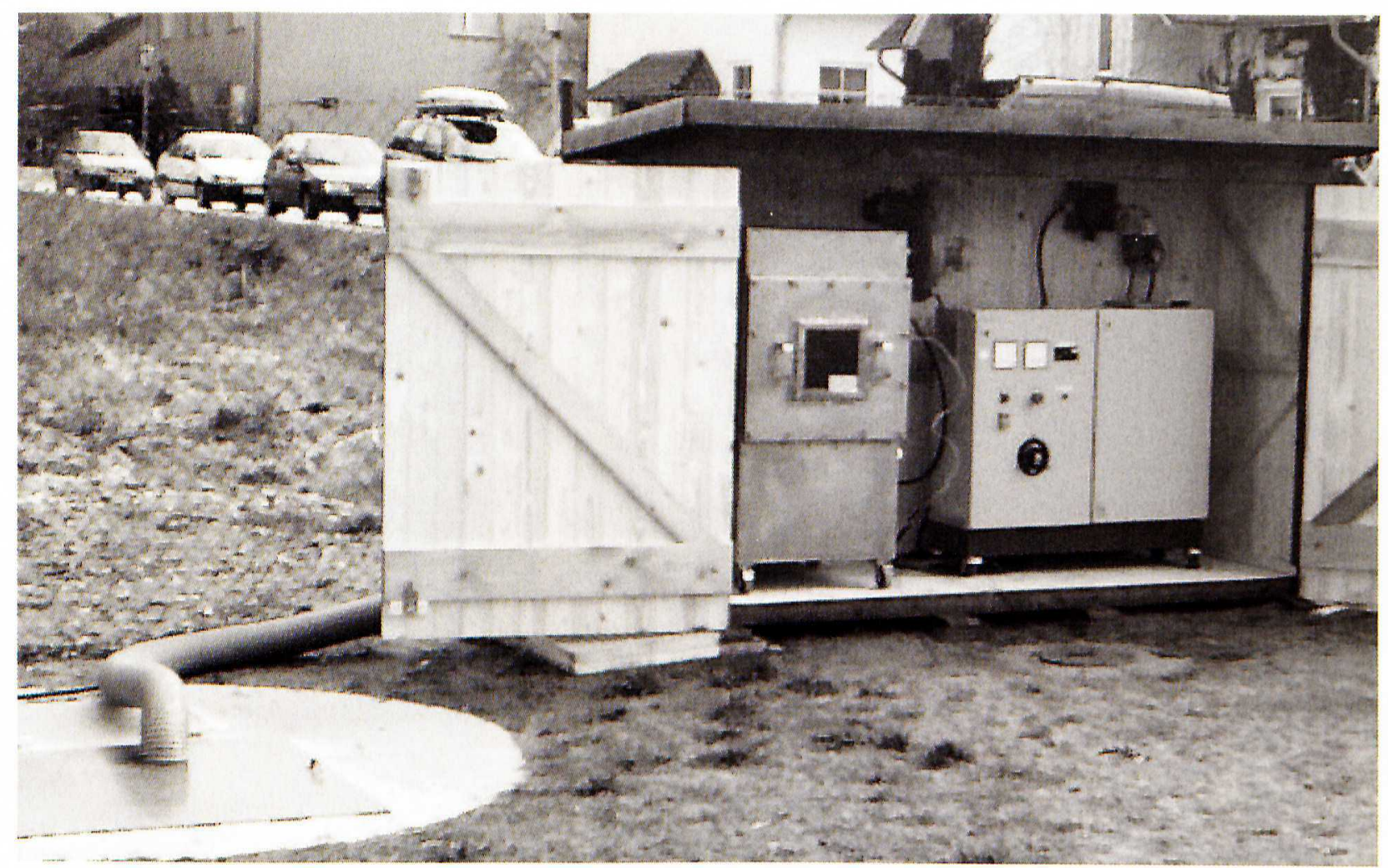

Plasmareaktor der TFH Wildau zur Geruchsbeseitigung an einem Abwasserpumpwerk der Dahme-Nuthe-Wasser-Abwasserbetriebsgesellschaft mbH 
Das IPLT ist zur Zeit mit dem Teilvorhaben „Experimentell gestuitzte Modellierung von Gasentladungen“ am Verbundvorhaben des BMBF: „Grundlagen der nichtthermischen Plasmachemie zur Abluftreinigung" beteiligt. Einer der Partner des Verbundprojektes ist das Institut für Umwelt Technologien GmbH, mit dem es seit vielen Jahren sehr enge Kooperationsbeziehungen gibt, was sich unter anderem in gemeinsamen Veröffentlichungen zeigt $[10,11]$. Auch dort haben schon Studenten der TFH Wildau Praktika absolviert.

Unsere Erfahrungen zeigen, dass das Ausbildungsprofil unserer Studenten sich an den Anforderungen der Forschung und Industrie orientiert. Die Zahl der Studenten, die fuir Praktika, Diplomarbeiten oder feste Anstellungen gesucht wird, ist aber bei weitem nicht ausreichend. Mit der Ausbildungsrichtung Plasmatechnik wird an der TFH Wildau in der Ausbildung von Ingenieuren Neuland betreten. Die Industrie im Umland, wie Rolls Roys Aeroengines, MTU und die Halbleiterindustrie in Frankfurt/ Oder, aber auch die Forschungsinstitute in Berlin und Brandenburg, werden verstärkt ihren Bedarf an gut ausgebildeten Ingenieuren anmelden.

\section{Literatur}

[1] BMBF Pubilk Plasmatechnik, 2000 oder http://www.bmbf.de

[2] Walter Weizel, Robert Rompe, Theorie elektrischer Lichtbögen und Funken, Leipzig 1949

[3] http://www.physik.uni-greifswald.de:8888/geschichte/ seeliger.html

[4] http://server-mei.physik.uni-greifswald.de/info/rutscher.html

[5] Rompe, R., \& Steenbeck, M. 1967, Ergebnisse der Plasmaphysik und der Gaselektronik, Band 1 (Akademie Verlag, Berlin)

[6] H. Gündel, Zustandssumme und effektive lonisierungsspannung eines Atoms im Plasma und die Möglichkeit ihrer experimentellen Überprüfung, Beiträge aus der Plasmaphysik, 10 (1970) 455 und 11 (1971) 1

[7] R. Rudolph, H.Beyer, Mikrowellenentladung für $\mathrm{CO}_{2}$-SlabLaser, Frühjahrstagung der Deutschen Physikalischen Gesellschaft, Erlangen 1994, 252

[8] H. Beyer, W. Bohmeyer, Annual Report 2000, Max-PlanckInstitut für Plasmaphysik, EURATOM Association, 161

(9] Harald Beyer, Werner Bohmeyer, Petra Kornejew, HansDieter Reiner, Gerd Fussmann, Comparison of Different Beam Sources for the Helium Beam Diagnostics, ECAMP VII, Berlin, 2001, P 12.16

[10] H. Beyer, H. Gündel, R. Rudolph, W. Ross, Vergleich von Elektronenstrahl und Impulskoronaentladung für die Zersetzung organischer Gase, 7. Bundesdeutsche Fachtagung für Plasmatechnologie, Bochum, 1996, 41

[11] D. Berger, H. Beyer, K.-P. Franck, W. Roß, W. Rudolph, Removal of Hydro- and Halocarbons from Off-Gases by Pulse Corona and Electron Beam Discharges, 12th international conference on gas discharges \& their applications, Greifswald 1997, 394

\section{Autoren}

Dipl. Phys. Harald Beyer

Dr.-Ing. Andreas Harendt

Karin Huget

Dr. Tobias Koschlig

Prof. Dr. rer. nat. Siegfried Rolle

E-Mail: rolle@pt.tfh-wildau.de

Dr. rer. nat. Werner Ross

Dipl.-Ing. Hans Volkmann

Technische Fachhochschule Wildau

Institut fuir Plasma- und Lasertechnik

Tel. (0 33 75) 508-126 